\title{
Revisão Sistemática e Meta-Análise da efetividade da reposição com testosterona em homens obesos com níveis séricos baixos de testosterona
}

Objetivo: avaliar a efetividade da reposição de testosterona para perda de peso e prevenção de complicações cardiovasculares em homens obesos com testosterona baixa. Métodos: foi realizada uma revisão sistemática de acordo com a Metodologia Cochrane de estudos randomizados que compararam em homens obesos com baixos níveis de testosterona a reposição de testosterona versus a não reposição. Dois revisores independentemente fizeram a seleção dos estudos e avaliaram o risco de viés dos estudos incluídos. Os desfechos primários foram: melhora dos parâmetros antropométricos (peso, índice de massa corpórea (IMC), circunferência de cintura e composição corporal), segurança, qualidade de vida, controle de complicações relacionadas a obesidade e melhora dos sintomas de hipogonadismo. As bases de dados eletrônicas foram Embase, Medline, LILACS e CENTRAL. A qualidade da evidência da foi gerada de acordo o GRADE. Resultados: 14 estudos foram incluídos. A meta-análise do peso final, IMC e circunferência da cintura não evidenciou diferença estatística entre os grupos (Diferença de Média (DM) -0.93, IC 95\%, -4.04 a 2.19, 446 participantes, 5 estudos; DM 0.42, IC 95\%, -0.41 a 1.24, 710 participantes, 7 estudos; DM -0.67, IC 95\%, -2.50 a 1.15, 692 participantes, 6 estudos, qualidade da evidência alta/moderada, respectivamente). A meta-análise não evidenciou um efeito claro da intervenção nos eventos cardiovasculares (RR 0.93, IC 95\%, 0.34 a 2.58, 562 participantes, 7 estudos, qualidade da evidência baixa), bem como para os desfechos qualidade de vida e controle metabólico (alta inconsistência entre os resultados dos estudos primários). Não houve diferença nos eventos adversos (RR 1.05, IC 95\%, 0.70 a 1.56, 624 participantes, 8 estudos, qualidade moderada). Conclusão: a reposição de testosterona em homens obesos não apresentou diferença significativa nos parâmetros antropométricos. Quanto aos eventos cardiovasculares, qualidade de vida, complicações relacionadas a obesidade e sintomas de hipogonadismo, o efeito da intervenção foi incerto. Registro PROSPERO: CRD42017065598. FAPESP 2018/11836-6 